\title{
About One Class of Representations of the Lie Algebra
}

\author{
M. HAVLÍČEK \\ Faculty of Mathematics and Physics, Charles University, Prague*
}

Received June 23, 1970

\begin{abstract}
Let $R(G)$ be the skewsymmetric representation of the algebra $G$ characterized by the following main property: if $G^{\prime} \subset G$ is some subalgebra of $G$ (possible noncompact) then $R\left(G^{\prime}\right)$ is integrable and reducible in the direct sum of irreducible representations of subalgebra $G^{\prime}$.

The paper is devoted to the development of the elementary theory of the described representations, culminating in the proof of one version of Schur's lemma.
\end{abstract}

\section{Introduction}

The skewsymmetric representations of the Lie algebra are usually met in connection with unitary representations of the corresponding group. From this point of view they have been investigated also by Nelson [1] who found condition of the construction of the unitary representation $R(\tilde{G})$ of the simply connected group $\tilde{G}$ from skewsymmetric representation $R(G)$ of its Lie algebra $G$-the condition of the integrability of $R(G)$.

So we have, in the case of infinitedimensional skewsymmetric representations, two essentially different classes of representations: integrable and nonintegrable. Because assumption of the integrability $R(G)$ does not always have a physical meaning, the nonintegrable representations of the Lie algebra can be interesting from a physical point of view [2].

Also the use of the infinitedimensional Lie algebras in theoretical physics leads to the study of the representations of the Lie algebra without limiting on the integrable ones [3,4]. In this case we do not usually have the corresponding group $\tilde{G}$ so that the concept of integrability of $R(G)$ loses its meaning.

Except for finitedimensional representations of the Lie algebras, the relatively simplest case arises for the semisimple ones, due to the results of Harish-Chandra [5]. If we denote $G^{\prime} \subset G$ the maximal compact

\footnotetext{
* Myslíkova 7, Prague 1.
} 
subalgebra of $G$, then $R\left(G^{\prime}\right) \subset R(G)$ is reducible in the direct sum of irreducible representations of $G^{\prime}$. The number $n_{i}$ of the representations equivalent to the representation $R_{i}\left(G^{\prime}\right)$ in this direct sum, is finite and the set of all such numbers has a finite upper bound. The representative Hilbert space $H$ may be written in the form

$$
H=\bigoplus_{i} H_{i} \otimes K_{i}
$$

where $H_{i}$ is representative finitedimensional Hilbert (i.e. unitary) space of the irreducible representation of $G^{\prime}$ (these representations are nonequivalent for $i \neq j$ ) and dimension of the unitary space $K_{i}$ equals $n_{i}$.

Due to these basic properties, the construction of the integrable skewsymmetric representations of the semisimple Lie algebras can be done by algebraic methods $[6,7]$.

This paper is devoted to the study of the skewsymmetric representations $R(G)$ of any Lie algebra $G$, characterized by the following main property. If $G^{\prime} \subset G$ is subalgebra of $G$ (possible noncompact), then $R\left(G^{\prime}\right)$ is integrable and reducible into the direct sum of the irreducible representations of $G^{\prime}$. If $G$ is semisimple and $G^{\prime}$ its maximal compact subalgebra we obtain the above mentioned representations of $G$.

Representations of this kind are met in the theory of elementary particles in connection with algebras coupling algebras of internal symmetries with the algebra of Poincaré (one particle representations) $[8,9]$.

Adding to the mentioned main property some mathematical assumptions defining the so called $G^{\prime}$-representations of $G$, we can construct these representations mostly in an algebraic way as in the case of semisimple $G$.

Note in this connection that in comparison with the wide possibilities given us by nonintegrable representations of Lie algebra, our mathematical assumptions restrict us to the relatively close class of representations described above. An interesting example of the more general representations of the certain Lie algebra is given in [15].

We start our considerations with basic definitions of the representation of the Lie algebra, its hermiticity and partial integrability. By the discussion of the possible domains of the representation (supports of representation) we come to the concept of partially analytical representation.

The second chapter is devoted to the definition of the discreteness of the representation with respect to some subalgebra $G^{\prime}$ and to the discussion of the concept of the $G^{\prime}$-irreducibility of these representations. An exact definition of the class of the representations of our interest is given (so called $G^{\prime}$-representations). 
The mathematical assumptions leading to the concept of $G^{\prime}$-representation were done mainly with respect to the proof of Schur's lemma which is the content of the last chapter.

\section{Basic Concepts}

At first we shall define, following [10], the representation of the Lie algebra $G$.

Definition 1. The representation $R(G)$ of the Lie algebra $G$ is homomorphism of the algebra $G$ into the set of linear operators in some Hilbert space $H$ so that:

a) there exists the dense linear subspace $D \subset H$, which is invariant with respect to all operators of $R(G)$ i.e. $R(x) D \subset D$ for every $x \in G$. Linear subspace $D$ will be called the support of $R(G)$. $f \in D$.

b) $R([x, y]) f=(R(x) R(y)-R(x) R(y)) f$ for every $x, y \in G$ and every

In the physical application of the representation $R(G)$, the essentially selfadjointness (e.s.a.) of the physically interpreted operators [11] is required: it means e.s.a. for some operators i $R(x), R(x) \in R(G)$. E.s.a. operators i $R(x)$ are symmetric i.e. $R(x)$ are skewsymmetric and this property we request, usually, from the rest operators of $R(G)$ on the support $D$, because the commutation relations connect all operators of $R(G)$ on the support $D$ and because the commutator of the skewsymmetric operators is skewsymmetric.

Definition 2. The representation $R(G)$ of the Lie algebra $G$ is called skewsymmetric if operators $R(x) \in R(G)$ are skewsymmetric for every $x \in G$.

The skewsymmetric representation $R(G)$ is called skewhermitian if operators i $R(x)$ physically interpreted are e.s.a.

The hermicity is not, however, the only restriction limiting the physical use of the skewsymmetric representations of $R(G)$ [2]. If we interprete generators of some subalgebra $G^{\prime} \subset G$ physically as the infinitesimal transformation in physical space and if we can experimentally verify the existence of the finite transformation (forming Lie group $\tilde{G}^{\prime}$ with Lie algebra $G^{\prime}$ ), we must restrict ourselves to such representations of $G$, that $R\left(G^{\prime}\right) \subset R(G)$ induces unitary representations of $\tilde{G}^{\prime}$. The representation $R(G)$ is then called partially integrable with respect to the subalgebra $G^{\prime} \subset G$.

Definition $3^{1}$. The skewsymmetric representation $R(G)$ with the support $D$ we call partially integrable with respect to the subalgebra $G^{\prime} \subset G$, if there exists the unitary representation $R\left(\tilde{G}^{\prime}\right)$ of the simply

1 This definition contains a wider class of representations than the definition of partially integrable ones in [2] and agrees with [16]. 
connected Lie group $\tilde{G}^{\prime}$ with Lie algebra $G^{\prime}$ such that the representation of $G^{\prime}$ derived from $R\left(\tilde{G}^{\prime}\right)$ equals to $R\left(G^{\prime}\right)$ on $D$.

If $G^{\prime}=G$ we call $R(G)$ integrable representation. The question of integrability of skewsymmetric representations of the Lie algebras was solved by Nelson [1] by use of the concept of the analytic vector ${ }^{2}$. Nelson showed that skewsymmetric representation $R(G)$ with the support $D$ is integrable if Nelson operator $\Delta=R^{2}\left(x_{1}\right)+\cdots+R^{2}\left(x_{d}\right)$ ( $x_{i}$-basis of the Lie algebra $G$ ) is e.s.a. (Theorem 5 of [1]).

Following Lemma 5.1 of the mentioned paper, the operator $A$ is e.s a. if and only if the set of analytic vectors of $A$ is dense in $H$.

Consider now the integrable representation $R(G)$ of the Lie algebra $G$ with support $D$. This representation gives rise to the unitary representation $R(\tilde{G})$ of the corresponding group $\tilde{G}$ which induces by differentiating representation $R^{\prime}(G) \supset R(G)$ of the algebra $G$.

As mentioned in [2], there exist two important dense linear subspaces of $H$ invariant with respect to the $R^{\prime}(G)$ which can be taken as the support of $R^{\prime}(G)$

a) $D_{\mathrm{an}}^{\Delta}$ - the set of all analytic vectors of the Nelson operator $\Delta$.

b) $D_{\text {an }}$ - the set of all analytic vectors of the representation $R(\tilde{G})^{3}$.

The connection of these two supports is given by inclusion $D_{\mathrm{an}} \supset D_{\mathrm{an}}^{\Delta}$ and every vector of $D_{\mathrm{an}}$ is also an analytic vector of every operator of $R(G)$.

It is seen that every integrable representation $R(G)$ induces representation $R^{\prime}(G)$ with support formed by the common analytic vectors of all operators $R(x) \in R(G)$. This fact motivate the following definition.

Definition 4. The representation $R(G)$ is called partially analytical with respect to $G^{\prime} \subset G$ if the support $D$ of $R(G)$ is formed by the common analytic vectors of the operators of $R\left(G^{\prime}\right)$.

For $G^{\prime}=G$ we call $R(G)$ analytical representation.

It is clear that a partially analytical representation with respect to subalgebra $G^{\prime}$ is partially integrable with respect to $G^{\prime}$. It follows immediately from the fact that vectors of $D$ are analytic vectors of Nelson operator of the subalgebra $G^{\prime}$. Further, the analytic representation is, at the same time, the representation hermitian, in the sense of the Definition 2. The concept of the partially analytic representation with respect to some subalgebra was introduced especially for the possibility of proving (under further assumptions) the version of Schur's lemma (see Chapter 3).

2 The vector $f \in H$ is called analytic vector of an operator $A$ if $\sum_{i=0}^{\infty} \frac{1}{i !}\left\|A^{i} f\right\| t^{i}<\infty$
for some $t>0$.

${ }^{3}$ The vector $f \in H$ is called an analytic vector of a representation $R(\tilde{G})$ if the mapping $g \rightarrow R(g) f$ of $G$ into $H$ is analytic in some neighbourhood of the identity.

10 Commun math. Phys, Vol. 20 


\section{II. $G^{\prime}$-Representations, Irreducibility}

The irreducibility of the finitedimensional representation of the Lie algebra $G$ is defined in the same way as the irreducibility of the representation of the Lie group. Because every representation of the Lie algebra is integrable in this case, the representation $R(G)$ of the Lie algebra $G$ is irreducible if and only if the representation $R(\tilde{G})$ of the corresponding Lie group $\tilde{G}$ with Lie algebra $G$ is irreducible too. In the case of infinitedimensional representations the situation is considerably complicated. Defining the irreducibility of the $R(G)$ in the usual way as a nonexistence of the nontrivial invariant closed subspaces of the representative Hilbert space $H$ (topological irreducibility), then integrable reducible representations $R(G)$ can induce the irreducible representations $R(\tilde{G})$.

As an example can serve the known Schrödinger representation of the algebra $[p, q]=i \cdot 1$ on the space $L^{2}(-\infty, \infty)$ by operators $p=d / d x$, $q=x$ with suitable support $D$. This representation is integrable in the irreducible (unitary) representation of the corresponding group and every $L^{2}(a, b) \subset L^{2}(-\infty, \infty)$ is invariant.

If we want a definition of irreducibility of $R(G)$ such that integrable irreducibile $R(G)$ shall lead to irreducible $R(\tilde{G})$ we must tolerate the existence of some invariant subspaces. This possibility is not on principle excluded, however it is simpler to define the irreducibility of the integrable $R(G)$ in the following way.

Definition 5. The integrable representation $R(G)$ of the Lie algebra $G$ is called irreducible if the representation $R(\tilde{G})$ is irreducible.

As to irreducibility of the partially integrable representations, we shall define this concept only for the class mentioned in the introduction ${ }^{4}$. At first we give their exact definition.

Definition 6. A skewsymmetric representation $R(G)$ we shall call discrete with respect to the subalgebra $G^{\prime} \subset G$ if

a) $R(G)$ will be analytical with respect to $G^{\prime}$

b) $R\left(\tilde{G}^{\prime}\right)=\bigoplus_{a} R_{a}\left(\tilde{G}^{\prime}\right)$ where $R_{a}\left(\tilde{G}^{\prime}\right)$ are irreducible representation of the group $\tilde{G}^{\prime}$.

If the several representations $R_{a}\left(\tilde{G}^{\prime}\right)$ equivalent to one representation is present in the mentioned decomposition of $R\left(\tilde{G}^{\prime}\right)$, this decomposition is not unique. If $\Omega_{b}(b=1, \ldots, p \leqq \infty)$ denotes the set of indices of the mutually equivalent representations in the decomposition of $R\left(\tilde{G}^{\prime}\right)$ and if

$$
R_{b}^{F}\left(\tilde{G}^{\prime}\right)=\bigoplus_{a \in \Omega_{b}} R_{a}\left(\tilde{G}^{\prime}\right),
$$

${ }^{4}$ For the possible definitions of the irreducibility of the general representations of the Lie algebras see [16]. 
then decomposition

$$
R\left(\tilde{G}^{\prime}\right)=\bigoplus_{b} R_{b}^{F}\left(\tilde{G}^{\prime}\right)
$$

is now unique. The corresponding decompositions of the representative Hilbert space is following

$$
H=\bigoplus_{b} H_{b}^{F} ; \quad H_{b}^{F}=\bigoplus_{a \in \Omega_{b}} H_{a} .
$$

With respect to the equivalence of the representations $R_{a}\left(\tilde{G}^{\prime}\right), a \in \Omega_{b}$ we can write

$$
H_{b}^{F}=H_{b} \otimes K_{b}
$$

where $K_{b}$ is the unitary space with the dimension equalled to the number of elements of $\Omega_{b}$. The consequence of Eq. (2) is the relation

$$
R_{b}^{F}\left(\tilde{G}^{\prime}\right)=R_{b}\left(\tilde{G}^{\prime}\right) \otimes I_{b}
$$

where $I_{b}$ is the identity operator on the space $K_{b}{ }^{5}$.

The irreducibility of the discrete representation $R(G)$ with respect to the subalgebra $G^{\prime}$ we shall understand essentially the impossibility of the reduction of $R(G)$ in the direct sum $R_{1}(G) \oplus R_{2}(G)$ of the representations of the same type. Simultaneously we must obtain a definition agreeing with Definition 5 for $G^{\prime} \equiv G$.

However, the following situation can arise without further additional assumptions. Consider the integrable reducible representation of the algebra $R(G)$ with support $D$. We can reduce $R(\tilde{G})$ in agreement with Definition 5 in the direct sum of representations and this reduction is not, in general, unique.

Let, therefore, $R(\tilde{G})=R_{1}(\tilde{G}) \oplus R_{2}(\tilde{G})$ be some reduction of $R(\tilde{G})$ and let $H=H_{1} \oplus H_{2}$ be corresponding decomposition of the representative space $H$. If $D \cap H_{a}$ will be not dense in $H_{a}$ for some $a=1,2$, then a corresponding reduction of $R(G)$ will not exist, in agreement with Definition 1, because $D \cap H_{a}$ does not have the properties of the support of representation of $G$. If this case would arise for all possible decomposition of $H, R(G)$ will be reducible (see Definition 5) but we shall not decompose $R(G)$ in the direct sum of the representations.

Therefore, we shall define the irreducibility of $R(G)$ for discrete $R(G)$ with the certain "minimal density" of the support $D$ only.

Definition 7. Let $R(G)$ be discrete representation of $G$ with respect to $G^{\prime} \subset G$. Let further, if $K$ is invariant subspace of the representation $R\left(\tilde{G}^{\prime}\right)$ then $K \cap D$ is dense in $K$. The representation $R(G)$ will be called $G^{\prime}$-irreducible if every subspace $K$ such that $R\left(\tilde{G}^{\prime}\right) K \subset K$ and $R(G)$ $(D \cap K) \subset K$ is trivial.

5 The same symbol used before sign of the tensor product will denote the identity operator on the space $H_{b}$. 
The definition has the following properties

a) It is equivalent, for $G^{\prime} \equiv G$, to the Definition 5 .

b) If $R^{\prime}(G) \supset R(G)$ (i.e. $D^{\prime} \supset D$ ) is further representation of the algebra $G$ then $G^{\prime}$-irreducibility of $R^{\prime}(G)$ is equivalent to the $G^{\prime}$-irreducibility of $R(G)$. To the proof it is sufficient to verify the following equivalence

$$
R(G)(D \cap K) \subset K \Leftrightarrow R^{\prime}(G)\left(D^{\prime} \cap K\right) \subset K .
$$

This equivalence is trivially fulfilled for $K \equiv H$. If $K \neq H$ we can write $H=K \oplus K^{\perp}$ and

$$
R(G)(D \cap K) \subset K \Leftrightarrow(R(G) f, g)=0
$$

for every $f \in D \cap K$ and every $g \in D \cap K^{\perp}$ because $D \cap K$ is dense in $K$ (analogical equivalence valid for $R^{\prime}(G)$ ). Let $f^{\prime} \in D^{\prime} \cap K$ and let $f_{n} \in D$, $f_{n} \rightarrow f^{\prime}$. Because operators of $R^{\prime}(G)$ are skewsymmetric and $R^{\prime}(G) \supset R(G)$ we have

$$
\left(R(G) f_{n}, g\right)=0 \Rightarrow\left(R^{\prime}(G) f^{\prime}, g\right)=\lim \left(f^{\prime}-f_{n}, R(G) g\right)=0
$$

for every $g \in D \cap K^{\perp}$ and therefore $R^{\prime}(G)\left(D^{\prime} \cap K\right) \subset K$. The proof of the equivalence in the second direction is trivial because $D^{\prime} \subset D$.

c) If $G_{1}^{\prime} \subset G_{2}^{\prime}$ are subalgebras of $G$ then we can easily prove that $G_{1}^{\prime}$-irreducibility of $R(G)$ implies $G_{2}^{\prime}$-irreducibility.

For the verification of the "minimal density" of the support $D$ of $R(G)$, we must know especially all invariant subspaces of the representation $R\left(\tilde{G}^{\prime}\right)$. The simplest way is to find the most general projector $E$ commuting with all operators of $R\left(\tilde{G}^{\prime}\right)$. Using results of [17] it is easily to prove that

$$
E=\bigoplus_{b} I_{b} \otimes E_{b}
$$

where $E_{b}$ is a projection on $K_{b}$. The set of all invariant subspaces of $R\left(\tilde{G}^{\prime}\right)$ we obtain from relation

$$
K=E H=\bigoplus_{b} H_{b} \otimes E_{b} K_{b}
$$

choosing projections $E_{b}$. We see that "minimal dense" support $D$ contains vectors $x_{b} \otimes f_{b}$ where $f_{b}$ is any vector $K_{b}$.

It is clear that an example of the "minimal dense" support is given by the following relation

$$
D=\sum_{b} D_{b} \otimes K_{b}{ }^{6}
$$

${ }^{6}$ We understand the symbol $\otimes$ in two slightly different ways. If $K$ and $H$ are closed linear spaces (i.e. Hilbert ones) then $K \otimes H$ means closed space under corresponding tensor product norm. In the remaining cases $K \otimes H$ is the linear space of all finite linear combinations of the vector $x \otimes y, x \in K, y \in H$. 
where $D_{b}$ is analytical support of the representation $R_{b}\left(G^{\prime}\right)$ and symbol $\sum$ denotes the algebraic direct sum. Support of this sort we shall use in the following.

The proof of the version of Schur's lemma presented later still lacks one additional assumption - the finitedimensionality of, at least, one space $K_{b}$. Due to this fact we limite ourselves by the stronger assumption, especially, $\operatorname{dim} K<\infty$ for all considered $b$, because it seems to be the most frequent case in practical applications. These representations we shall call in brief $G^{\prime}$-representations.

Definition 8. The $G^{\prime}$-irreducible representation $R(G)$ with support given by Eq. (3) such that $\operatorname{dim} K_{b}<\infty$ for any $b$ will be called $G^{\prime}$ - representation.

\section{Two Theorems}

Lemma. Let $R_{1}(G)$ and $R_{2}(G)$ be irreducible analytical representations of the algebra $G$ on the spaces $H_{1}$ and $H_{2}$ with supports $D_{1}$ and $D_{2}$. Let $S_{21}$ and $S_{12}$ be two mappings of $H_{1}$ into $H_{2}$ into $H_{1}$ respectively with the following properties

1) $S_{21} D_{1} \subset D_{2}, \quad S_{12} D_{2} \subset D_{1}$,

2) $\left(S_{12} f, g\right)=\left(f, S_{21} g\right), \quad f \in D_{2}, g \in D_{1}$,

3) $\left(S_{12} R_{2}(G)-R_{1}(G) S_{12}\right) D_{2}=0$, $\left(S_{21} R_{1}(G)-R_{2}(G) S_{21}\right) D_{1}=0$.

Then either $S_{12} f=S_{21} g=0$ or $R_{1}(\tilde{G})$ and $R_{2}(\tilde{G})$ are unitarily equivalent, $R_{2}(\tilde{G})=U^{-1} R_{1}(\tilde{G}) U$, and $S_{12} f=\lambda \cdot U f, S_{21} g=\lambda^{-1} U^{-1} g$ for any $f \in D_{2}, g \in D_{1}$ where $\lambda$ is a real number.

Proof. At first we prove this lemma for $R_{1}(G) \equiv R_{2}(G) \equiv R(G)$, $D_{1} \equiv D_{2} \equiv D, S_{12} \equiv S_{21} \equiv S$ and $H_{1} \equiv H_{2} \equiv H$. We start with the construction of the unitary operator $\bar{V}$. The Caley transformation of the operator $S$

$$
V=(S+i I)(S-i I)^{-1}
$$

is isometric mapping of the linear subspace $D_{-}=(S-i I) D \subset D$ on the linear subspace $D_{+}=(S+i I) D \subset D$. Let $f_{+}$be any vector of $D_{+}$. Then there exists at least one vector $f \in D$ so that $f_{+}=(S+i I) f$ and

$$
R(x) f_{+}=(S+i I) R(x) f \in D_{+}
$$

for every $x \in G$ because of $R(x) f \in D$. Therefore subspace $D_{+}$is invariant subspace of $R(G)$ and in agreement with [5], $\bar{D}_{+}$is invariant subspace of $R(\tilde{G})$, because $D_{+} \subset D$ is formed by analytic vectors of $R(G)$. Due to irreducibility of the representation $R(G)$ we have $\bar{D}_{+} \equiv H$ and denseness of $D_{+}$is proved. As a consequence of this property of $D_{+}$we can extend the operators $V$ to unitary operator $\bar{V}[10]$. 
From commutativity of the operator $S$ with operators $R(G)$ and from skewsymmetricity of $R(G)$ we easily prove

$$
(\bar{V} \overline{R(x)} f, g)=-(\bar{V} f, \overline{R(x)} g)
$$

for any $f \in D_{-}, g \in D_{+}$and any $x \in \mathrm{G}$.

We prove the validity of this equation for any analytic vectors $f, g$ of $\overline{R(x)}$. Denote by $r_{-}$and $r$ the restrictions of the operator $R(x)$ ( $x$-fixed) to the domains $D_{-}$or $D$ respectively. The closures of these operators are skewadjoint i.e.

$$
\bar{r}_{-}=-\left(\bar{r}_{-}\right)^{*}, \quad \bar{r}=-(\bar{r})^{*}
$$

because vectors of $D_{-}$and $D$ are analytic vectors of $\bar{r}_{-}$or $\bar{r}$ respectively. Using this fact and trivial implications

$$
r_{-} \subset r \Rightarrow \bar{r}_{-} \subset \bar{r} \Rightarrow \bar{r}^{*} \subset \vec{r}_{-}^{*}
$$

we obtain $\bar{r}_{-}=\bar{r}$. It means, especially, that for any analytic vector $f$ there exists a sequence $f_{n} \in D_{-}, f_{n} \rightarrow f$ so that $R(x) f_{n} \rightarrow \overline{R(x)} f$.

Substituting $f$ in Eq. (5) by $f_{n}$ and limitating we extend this equation to any analytic vector $f$ of $\overline{R(x)}$. The same arguments can be used for extension to any analytic vector $g$ of $\overline{R(x)}$.

From the Eq. (5) we obtain further the relation

$$
\left(\bar{V}\left(\sum_{k=0}^{n} \frac{1}{k !}(\overline{R(x)})^{k} t^{k}\right) f, g\right)=\left(\bar{V} f,\left(\sum_{k=0}^{n} \frac{1}{k !}(-\overline{R(x)})^{k} t^{k}\right), g\right)
$$

valid for any natural $n$, any $t>0$ and any analytic vectors $f, g$ of $\overline{R(x)}$.

Due to this property, the sums inside of scalar product converge for some $|t|<s$ (s may depend on $f, g$ and $x \in G$ of course) to $\exp \overline{R(x)} t \cdot f$ or $\exp (-\overline{R(x)} t) \cdot g$ respectively, so that we have

$$
(\bar{V} \exp \overline{R(x)} t \cdot f, g)=(V f, \exp (-\overline{R(x)} t) \cdot g)
$$

for $|t|<s$. As it is shown in [1] (the proof of Lemma 5.1, p. 584) the vector $\exp \overline{R(x)} t \cdot f$ is analytic vector of $\overline{R(x)}$ again and radius of convergence of the corresponding sum is not less as in the case of vector $f$. The consequence of this fact is the validity of the relation (6) for any $t$. Actually, we can write

$$
\exp \overline{R(x)} t=\left(\exp \overline{R(x)} \frac{t}{m}\right)^{m}
$$

where natural number $m$ is choosen so that $|t / m|<s$ and proof is completed by $m$-times use of the above relation.

Therefore, Eq. (6) holds for any $t$ and any $f, g \in D$ so that we can extend its validity to all vectors of $H$. Now this equation gives

$$
[\bar{V}, \exp \overline{R(x)} t]=0 .
$$


If elements $x t \in G$ are sufficiently near to origin, then operators $\exp \overline{R(x)} t$ realize the unitary representation $R_{U}(\tilde{G})$ of some neighbourhood $U$ of identity of the group $\tilde{G}$. Because of simple connectness of the group $\tilde{G}$ there exists the unique extension of $R_{U}(\tilde{G})$ in the representation of the group $\tilde{G}$ on space $H$ and therefore the operator $\bar{V}$ commutes with all operators of $R(\tilde{G})$. Using the usual Schur's lemma we obtain $\bar{V}=a I$ where $a$ is, due to unitarity of $\bar{V}$, a complex unit i.e. $|a|=1$. By reversion of the Caley transformation (1) of the operators $S$ we obtain

$$
S f=\lambda f
$$

where $\lambda=\mathrm{i}(a+1)(a-1)^{-1}$ is a real number.

Now we prove the general assertion of the lemma. The operator $S_{12} \cdot S_{21}$ mapping $H_{1}$ into $H_{1}$, commute with the representation $R_{1}(G)$ on the support $D_{1}$ and fulfils all assumptions of the just proved specified assertion of lemma so that $S_{12} \cdot S_{21}=\lambda I$ on $D_{1}$. From the second assumption of lemma we have

$$
\left\|S_{21} f\right\|^{2}=\left(S_{21} S_{12} f, f\right)=\lambda\|f\|^{2}
$$

for any $f \in D_{1}$ which showes that $\lambda \geqq 0$.

If now $\lambda=0$ then $S_{21} f=0$ for any $f \in D_{1}$ and therefore $S_{12} g=0$ for any $g \in D_{2}$ (again see second assumption of lemma).

If $\lambda>0$ we can extend, at first, the mapping $S_{21}$ to the bounded mapping $\bar{S}_{21}$. As it is seen from Eq. (7), this mapping is, up to multiplicative constant, the isometry mapping of $H_{1}$ onto $H_{2}$. Further we can rewrite the second part of the third assumption of the lemma in the equivalent form

$g \in D_{1}, f \in D_{2}$.

$$
\left(\bar{S}_{21} R_{1}(x) g, f\right)=-\left(\bar{S}_{21} g, R_{2}(x) f\right)
$$

From this relation we easily derive, repeating the procedure of the above proof, the equation

$$
\bar{S}_{21} R_{1}(\tilde{G})-R_{2}(\tilde{G}) \bar{S}_{21}=0
$$

and assertion of the lemma we now obtain quite trivially.

Theorem 1. Let $R(G)$ be a discrete representation of $G$ with respect to $G$ with the support $D \subset H=\bigoplus_{b} H_{b} \otimes K_{b}$ (see Eqs. (1-2)) and let $D^{\prime}=\sum_{b} D_{b} \otimes K_{b}^{\prime} \subset D$ where $K_{b}^{\prime}$ is some dense linear subspace of $K_{b}$. Let further $S$ be symmetric operator on the space $H$ with the following properties.

1) $S D^{\prime} \subset D^{\prime}$

2) $[S, R(G)] D^{\prime}=0$. Then for any $f \in D^{\prime}, S f=\left(\bigoplus_{b} I_{b} \otimes S_{b}\right) \cdot f$, where $S_{b}$ denotes any symmetric operator on $K_{b}$ with the domain $D\left(S_{b}\right) \supset K_{b}^{\prime}$. 
Proof. It is sufficient to prove the assertion of the theorem for vectors of the form $f_{b} \otimes e_{b}, f_{b} \in D_{b}, e_{b} \in K_{b}^{\prime}$ because the subspace $D^{\prime}$ is their linear span.

Let $b^{0}$ be fixed and take any $f_{b^{0}} \in D_{b^{0}}$ and any $e_{b^{0}} \in K_{b^{0}}^{\prime}$. With respect to the separability of all $K_{b}$ we can construct an orthonormal countable basis $\left\{e_{z b}\right\}, z=1, \ldots, \operatorname{dim} K_{b}$ for any $K_{b}$ in such a way that $e_{b^{0}} \equiv e_{1 b^{0}}$ and $e_{z b} \in K_{b}^{\prime}$. Now we can write

$$
S f_{b} \otimes e_{z b}=\sum_{z^{\prime}, b^{\prime}}\left(S_{z b}^{z^{\prime} b^{\prime}} f_{b}\right) \otimes e_{z^{\prime} b^{\prime}}
$$

where $S_{z b}^{z^{\prime} b^{\prime}}$ are mappings of the space $H_{b}$ into $H_{b^{\prime}}$ which maps $D_{b}$ into $D_{b^{\prime}}$, and assumption 2) immediately gives

$$
\left(S_{z b}^{z^{\prime} b^{\prime}} R_{b}(G)-R_{b^{\prime}}(G) S_{z b}^{z^{\prime} b^{\prime}}\right) D_{b}=0 .
$$

From the symmetry of the operator $S$ we easily find that mappings $S_{z b}^{z^{\prime} b^{\prime}}$ and $S_{z b}^{z^{\prime} b}$ fulfil also the second assumption of the preceding lemma and therefore we can use its assertion for these mappings.

So we obtain

$$
S_{z b}^{z^{\prime} b^{\prime}}=\delta_{b b^{\prime}} \lambda_{z z^{\prime}} I
$$

on $D_{b}$, because representations $R_{b}(\tilde{G})$ are nonequivalent for different $b$. Substituting it in Eq. (8) we obtain

where

$$
S f_{b} \otimes e_{z b}=f_{b} \otimes S_{b} e_{z b}
$$

$$
S_{b} e_{z b}=\sum_{z^{\prime}} \lambda_{z z^{\prime}} e_{z^{\prime} b} .
$$

Because we start our considerations with any $f_{b^{0}} \otimes e_{b^{0}} \equiv f_{b^{0}} \otimes e_{1 b^{0}}$ these relations shows the validity of the assertion of theorem for any $f \in D^{\prime}$.

Theorem 2. (Schur's lemma). Let $R(G)$ be a $G^{\prime}$-representation of $G$ with support $D \subset H$. Let $S$ be symmetric operator with the following properties

1) $S D \subset D$

2) $[S, R(G)] \cdot D=0$.

Then $S f=\lambda f$ for any $f \in D$ and $\lambda$ is a real number.

Proof. The operator $S$ fulfils the assumptions of the preceeding theorem and therefore $S=\bigoplus_{b} I_{b} \otimes S_{b}$ on $D$. Because all spaces $K_{b}$ are finitedimensional (see Def. 8), all $S_{b}$ can be diagonalized i.e. there exists such basis $\left\{e_{z b}\right\} z=1, \ldots, \operatorname{dim} K_{b}$ in the spaces $K_{b}$ that $S_{b} e_{z b}=\lambda_{z b} e_{z b}$. Denote by $H_{\lambda}$ the direct sum of all spaces $H_{b} \otimes e_{z b}$ such that $\lambda_{z b} \equiv \lambda$. 
This subspaces is, due to commutativity of the operators of $R(G)$ with $S$, invariant subspace of $R(G)$. If there exist at least two different eigenvalues $\lambda \neq \lambda^{\prime}$ then $H_{\lambda} \neq H$ which is in contradiction with $G^{\prime}$-irreducibility of $R(G)$. Therefore $S_{b} e_{z b}=\lambda e_{z b}$ for any $z$ and $b$ so that $S f=\lambda f$ for any $f \in D$.

\section{Concluding Remarks}

a) The two fundamental mathematical assumptions applied to the discrete representation $R(G)$ with respect to $G^{\prime}$ are partial analyticity of $R(G)$ with respect to $G^{\prime}$ and especial form of the support $D$ (see Eq. (3)). The first assumption identifies the support $D$ of $R(G)$ with linear subspace of the common analytic vectors $D_{\text {an }}^{\prime}$ of the operators of $R\left(G^{\prime}\right)$ which is not, in general, necessary. These assumptions, however, make it possible to prove the version of Schur's lemma on the basis of the algebraic property only - symmetricity of the commuting operator - without such additional assumptions as boundedness of $S$ etc.

b) The change of the form of the support $D$ given by Eq. (3) can lead to further interesting representations of $G$. It best illustrates, as can be easily proved, the example of nonintegrable representation, of such kind, of the finitedimensional Lie algebra containing Poincaré algebra and giving the nontrivial discrete mass spectrum discussed in [15].

c) If $S$ in Theorem 2 is bounded then $S=\lambda I$ on whole $H$. It shows that our $G^{\prime}$-irreducibility of $R(G)$ implies Schur's irreducibility of $R(G)$ introduced in [16].

d) It is clear that the main part of our preceeding considerations in Chapters 1-3 holds also for infinitedimensional $G$ and finitedimensional subalgebra $G^{\prime}$.

\section{References}

1. Nelson, E.: Ann. Math. 70, 572 (1959).

2. Doebner, H. D., Melsheimer, O.: Nuovo Cimento 49, 73 (1967).

3. Formánek, J.: Nucl. Phys. 87, 376 (1966).

4. Votruba, J., Havliček, M.: In: High energy physics and theory of elementary particles, p. 330. Kiev: Naukova dumka 1967.

5. Harish-Chandra: Trans. Am. Math. Soc. 75, 185 (1953).

6. Gelfand, I. M., Minlos, P. A., Shapiro, Z. J.: Representations of the rotation and Lorentz group. London: Pergamon Press 1963.

7. Dixmier, J.: Bull. Soc. Math. Franc. 89, 9 (1961).

8. Havliček, M., Votruba, J.: Czech. J. Phys. B 16, 631 (1966).

9. Mukunda, N., Sudarshan, E. C. G., Böhm, A.: Phys. Letters 24 B, 301 (1967).

10. Maurin, K.: Methods of Hilbert spaces. Moscow: Izd. Mir. 1965 (in Russian).

11. Mackey, G. W.: Lessons on the mathematical foundations of quantum mechanics. Moscow: Izd. Mir. 1965 (in Russian). 
12. Wigner, E. P.: Ann. Math. 40, 139 (1939).

13. Širokov, Ju. M.: Exp. Theor. Phys. 33, 861 (1957).

14. Havliček, M.: About representations of the certain infinite-dimensional Lie algebra. Candidate dissertation, Fac. of Math. and Phys. of the Charles University in Prague. December 1968 (in Czech).

15. Flato, M., Sternheimer, D.: Poincaré partially integrable local representations and mass-spectrum. Preprint of Physique Mathématique, Collége de France. November 1968 (to be published in Commun. Math. Phys.)

16. Sternheimer, D.: Note sur les représentations locales d'algebras de Lie. Preprint of Physique Mathématique, Collége de France.

17. Najmark, M. A.: Normed rings. Moscow: Gostěchizdat 1956 (in Russian).

\author{
M. Havlíček \\ Dept. of Theoretical Physics \\ Faculty of Mathematics a. Physics \\ Charles University \\ Myslíkova 7 \\ Prague 1, CSSR
}

